IMAGES IN MEDICINE.

\title{
Facial and dental appearance of Williams syndrome
}

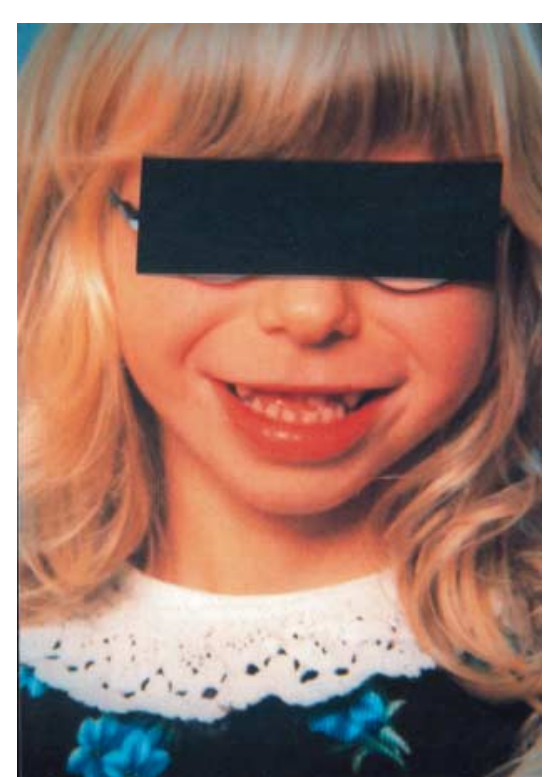

Figure 1 Typical facial appearance of a Williams syndrome patient showing wide mouth with long phyltrum and thick lips (published with parent's permission).

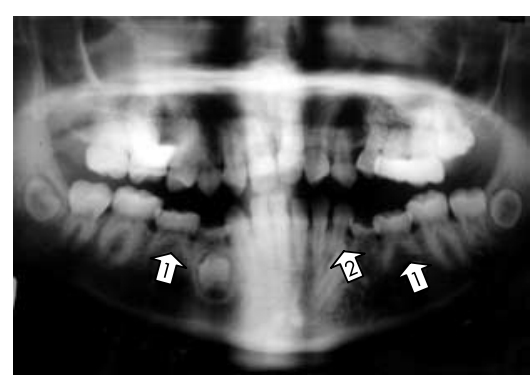

Figure 2 Orthopantomogram tomographic radiography of a typical Williams syndrome patient showing aplasia and tooth resorption anomaly, with fan shaped positioning of the front teeth.
1 illiams syndrome is a genetic condition, which predominantly occurs as a sporadic disorder, although some families have been reported showing autosomal dominant inheritance with varying penetrance. The incidence of Williams syndrome is estimated to be one in 20 000-50 000 live births, and it consists of supravalvular aortic stenosis, characteristic dysmorphic facial features named "elfin face" (wide mouth with long phyltrum and thick lips; fig 1), mental retardation, and other clinical manifestations including transient infantile idiopathic hypercalcaemia, growth retardation, and frequent dental problems. ${ }^{1}$ The syndrome is caused by a submicroscopic deletion in the chromosome 7 implicating the $7 \mathrm{q} 11.23$ region (elastic gene). ${ }^{2}$ Early diagnosis of the syndrome is important since many of its features require treatment, and the prognosis can be dramatically improved by timely management. The clinical complexity of this developmental disorder is well known, making it difficult to diagnose on the clinical picture, while genetic testing is expensive and it is not cost effective to screen all patients. A dentist can be of help in diagnosing Williams syndrome early.

We examined 33 children with Williams syndrome, proved by fluorescent in situ hybridisation at the pedodontic and orthodontic department of Semmelweis University in Budapest, Hungary. We found that typical dental findings in Williams syndrome are dental aplasia, which occurred in $90 \%$ of our patients, and primary tooth resorption anomaly, which we found in $96 \%$ of our patients. Also, fan shaped positioning of the front teeth was seen in the majority of our patients (fig 2). We highlight the fact that early orthodontic consultation is very important to avoid further dental anomalies in Williams syndrome.

I Tarjan, G Balaton, P Balaton, S Varbiro*, Z Vajo Semmelweis University, Department of Dentistry for Children and Orthodontics and 2nd Department of Obstetrics and Gynaecology*, Mária st 39, 1085 Budapest, Hungary; vzoltan@asu.edu

\section{References}

1 Onis Vilches MC, Rubio Cuadrado MV, Martinez de la Iglesia J, et al. William's syndrome. Report of a case with family involvement. Rev Clin Esp 1998;198:91-4.

2 Mila M, Carrio A, Sanchez A, et al. Clinical characterization, molecular and FISH studies in 80 patients with clinical suspicion of Williams-Beuren syndrome. Med Clin (Barc) 1999;1 13:46-9. 